\title{
THE EFFECT OF RECTANGULAR SYNTHETIC JET TO THE FLAT PLATE BOUNDARY LAYER IN LOW REYNOLDS NUMBER
}

\author{
Young-Hwan KIM \\ Aeronautical engineering, Emirates Aviation College, Dubai, United Arab Emirates
}

\begin{abstract}
The objective of this study is investigating the impact of synthetic jet on the boundary layer and optimising the slot yaw angle $(\beta)$. A rectangular synthetic jet with yaw angle relative to freestream is applied for flat plate boundary layer control. Various slot yaw angles are applied to find optimized amount and their impacts on the boundary layer are measured using a boundary layer probe with 2-D traversing system. The slot yaw angles are varied from $0^{\circ} \sim 90^{\circ}$. The assessment carried out using non-dimensional velocity profiles and lateral relative velocity $(\mathrm{u} / \mathrm{U})$ contours.
\end{abstract}

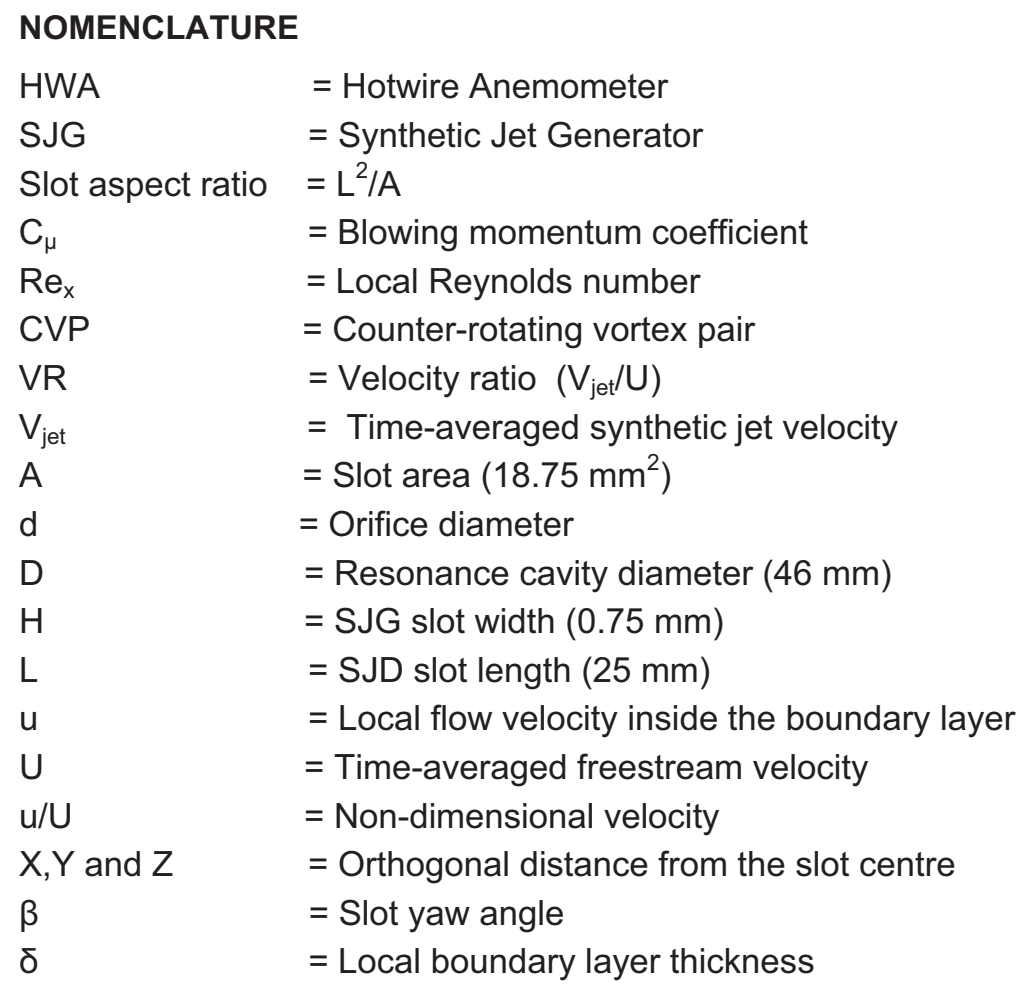

\section{INTRODUCTION}

Synthetic jet is created by the cyclic volume change of internal cavity, which is connected to surrounding air through an orifice. There are many bidirectional pumping devices, which can create high frequency internal volume change for synthetic jet generator (SJG) and the piezoelectric diaphragm is selected in this study. Xin zhang et al. ${ }^{[1]}$ presented that the yaw angle of steady jet relative to freestream is able to create streamwise

\footnotetext{
- Assistant professor, Aeronautical Engineering, Emirates Aviation College, Dubai, United Arab Emirates, yhkim@emirates.com
}

This is an Open Access article distributed under the terms of the Creative Commons Attribution License 2.0, which permits unrestricted use, distribution, and reproduction in any medium, provided the original work is properly cited. 
vortex and Bridges et al. ${ }^{[2]}$ presented that synthetic jet with rectangular orifice is able to create it too. Since the streamwise vortex generation using a rectangular slot with yaw angle is the boundary layer control scheme in the following study, the rectangular synthetic jet is investigated.

\section{Design of Piezoelectric-driven Synthetic Jet Generator}

Basically, the piezoelectric diaphragm is a metal plate, which is adhered by a piezoelectric ceramic on one face. Piezoelectric ceramic is a unique material, which expands and shrinks its planar area with the change of applied electric field direction. On the contrary, metal plate does not change its area regardless of electric field. Therefore high frequency AC voltage will cause the vibration of piezoelectric diaphragm due to the difference of deformation ratio between these materials, see Figure 1. A 7BB-50M-1 piezoelectric diaphragm of Murata $\mathrm{Co}^{[5]}$ is applied for SJG fabrication in this study. Basically this diaphragm is designed for acoustic device and $50 \mathrm{~mm}$ diameter nickel-plated brass is adhered to $25 \mathrm{~mm}$ diameter piezoelectric ceramic.

A single diaphragm low- $\mathrm{C}_{\mu}$ SJG was developed for this study. This SJG has a modular design and is composed of 5 parts as illustrated in Figure 2 and assembled by 4 screws, which are evenly distributed around the resonance cavity. Every screw is carefully torqued to provide homogeneous clamping force around the resonance cavity. During the preliminary study, the author recognised that the clamping force affects the resonance frequency and resulting synthetic jet velocity. Because leakage of air through the gap between parts can deteriorate the performance of SJG, sealing material was applied to every part during the assembly procedure. The SJG has a square body with a cylindrical resonance cavity. The resonance cavity diameter (D) is determined as $46 \mathrm{~mm}$ to allow $2 \mathrm{~mm}$ of the boundary for clamping. The geometry of SJG was determined by a preliminary quick HWA experiment. Figure 3 illustrates the determined slot dimensions and the relative slot aspect ratio is 33.3. Every part of the generator is fabricated in brass.

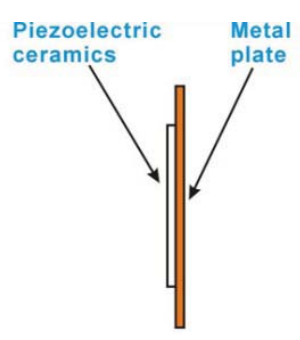

(a) Steady

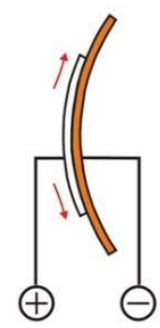

(b) Extension (DC voltage)

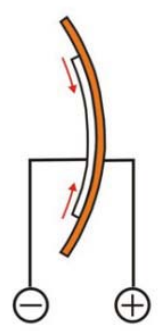

(c) Shrink (DC voltage)

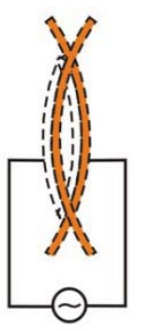

(d) Vibration (AC voltage)

Figure 1: The principle of piezoelectric diaphragm oscillation ${ }^{[5]}$ 


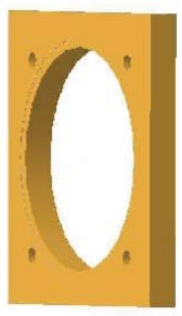

Mounting element

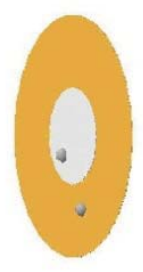

Piezoelectric diaphragm

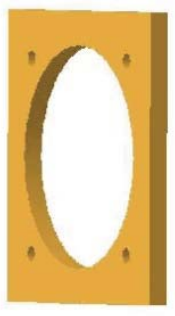

Cavity element

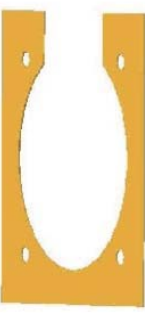

Slot element

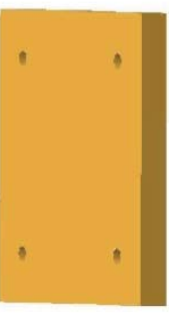

Base element

Figure 2: The structure of SJG

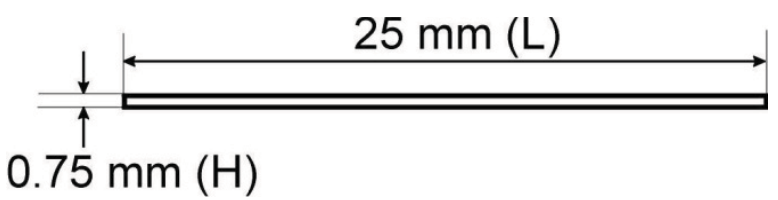

Figure 3: Dimension of synthetic jet slot

\section{Experimental ARRAngement}

The experimental arrangement is composed of SJG driving system (The function generator \& amplifier) and synthetic jet measurement system. A Thander TG 2002 function generator was used to generate maximum $5 \mathrm{~V}_{\mathrm{p}-\mathrm{p}}$ excitation signal. This signal was fed into Ariston AX-910 integrated amplifier, amplified to $50 \mathrm{~V}_{\mathrm{p}-\mathrm{p}}$ and used to excite the SJG. The strength of the synthetic jet is able to be controlled by changing the excitation frequency and amplitude. Since the relation between the excitation frequency and the synthetic jet velocity is highly nonlinear, an initial calibration of the resonance characteristics is necessary to ensure optimum performance of the SJG.

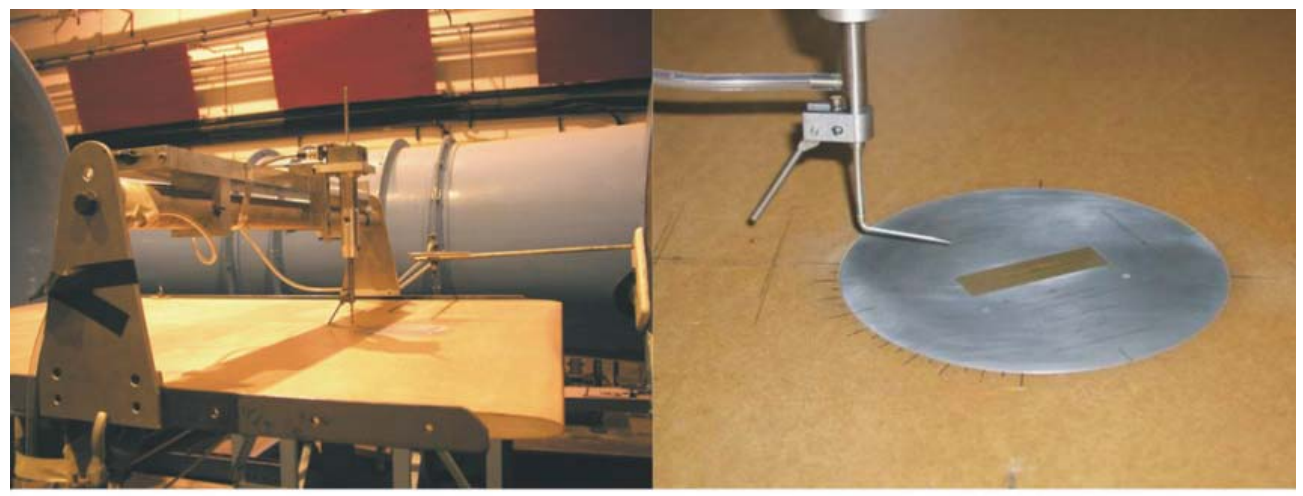

(a) Traversing system

(b) Boundary layer probe

Figure 4: Boundary layer measurement systems (Total pressure measurement) 
Figure 4 presents the instrumentation for boundary layer measurements on the flat plate. The flowfield survey is carried out using a boundary layer probe. The ruggedness of the boundary layer probe is more convenient than a fragile HWA probe. The definition of flat plate coordinate system is illustrated in Figure 5. Because the centre of slot is defined as the origination of flat plate coordinate, the upstream area of slot is expressed by negative sign $(-X)$.

A 2-D traverse system was used for the non-dimensional velocity ( $u / U$ ) distribution survey. Vertical traversing (Z-direction) is carried out automatically using software driven motor. Lateral traversing (Y-direction) has to be conducted manually, for the longitudinal (streamwise) movement of boundary layer probe (X-direction), the entire traversing system on the lateral support is relocated. The synthetic jet turntable is rotated manually for the investigation of slot yaw angle effect.

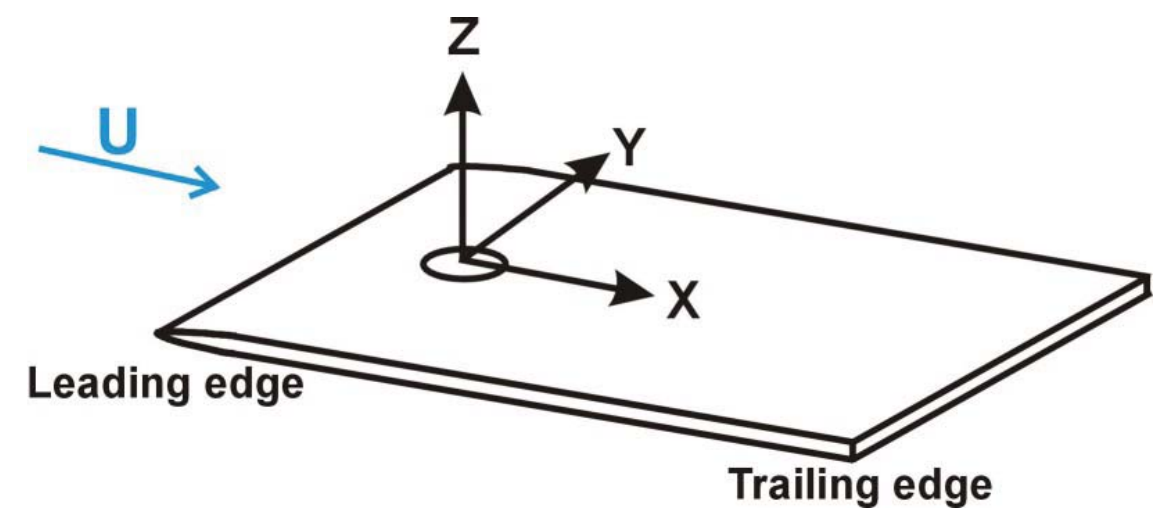

Figure 5: Definition of flat plate coordinate system

Two power supply units of Farnell instrument LTD is used for the driving of boundary layer probe traverse system. The total pressure, which is acquired by boundary layer probe, is fed into the Furness Controls Limited, FCO 44 bi-directional differential pressure transducer, which covers ranges \pm 20 Pascals. The reference total pressure and static pressure are acquired by the Pitot-static tube, which is installed near to the boundary layer probe. The static pressure data from Pitot static prove is supplied to both ch0 and ch1 of FCO 44 for freestream velocity $(U)$ and local flow velocity inside boundary layer (u) calculation. The reference atmospheric pressure data are achieved by DPI 141 resonant sensor barometer and supplied to the data acquisition control box. Figure 6 presents the detail of flat plate model, which used for this study. The boundary layer probe have flat mouth and its closest proximity to the surface is $0.1 \mathrm{~mm}$, see Figure 7 . The Pitot-static tube data, barometer and boundary layer probe data are linked to the data acquisition control box, which is connected to the A/D board of control computer. 

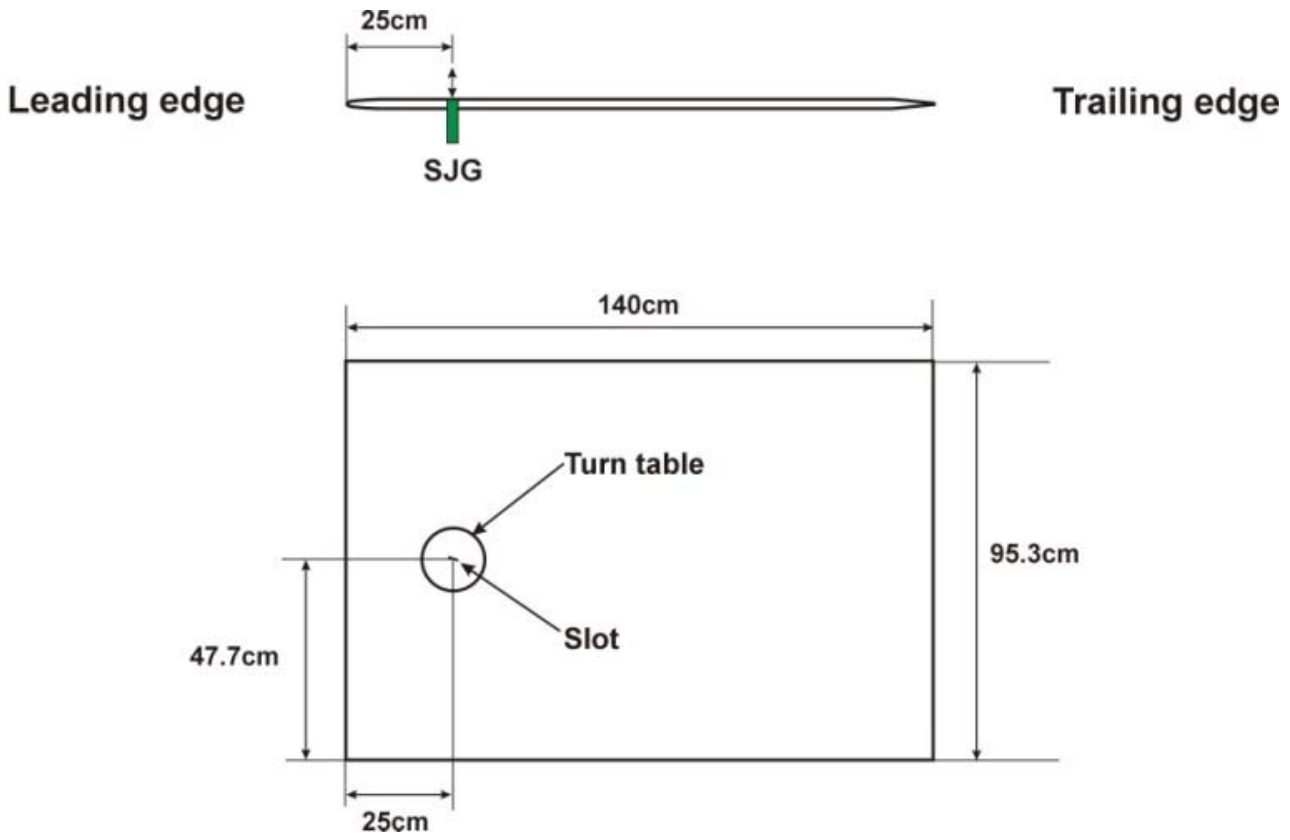

Figure 6: Flat plate model

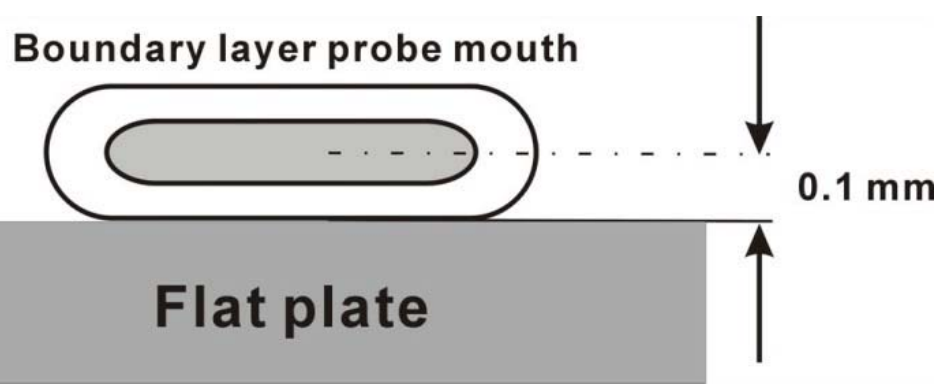

Figure 7: The closest proximity of boundary layer probe mouth to the flat plate

When using a steady jet with rectangular slot, the strength of the longitudinal vortices can be controlled by the changing of the slot yaw angle $(\beta)$. The size and strength of the resulting longitudinal vortex, its rotational direction, and consequently the flow control effectiveness of the device, strongly depends on the slot yaw angle. ${ }^{[2]}$ As can be seen in Figure $8, \beta$ is defined as $0^{\circ}$, when the longitudinal-axis $(X)$ of the slot is in line with the freestream direction, and counter-clockwise rotation of the turntable is defined as a positive yaw $(+\beta)$. All the measurements to slot yaw angle effectiveness were made with the excitation frequency of SJG fixed at its acoustic resonance frequency $(970 \mathrm{~Hz})$. A turntable with SJG is mounted in the flat plate. The SJG is flush-mounted to the turntable to avoid triggering laminar-turbulent transition. However, the following experimental data presents that the mounting of this turntable was not perfect. 


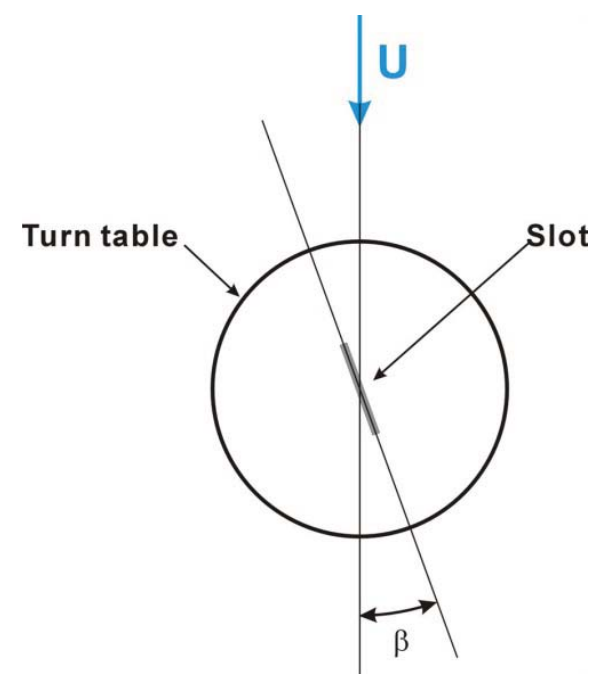

Figure 8: Definition of slot yaw angle $(\beta)$ relative to freestream direction

Figure 9 shows the coordinate system and dimension of the SJG slot, which were used to define non-dimensional parameters and locations for this study. The longitudinal distance between the centre of the slot $(X=0 \mathrm{~mm})$ and the boundary layer probe tip is marked in Figure $\mathbf{1 0 .}$
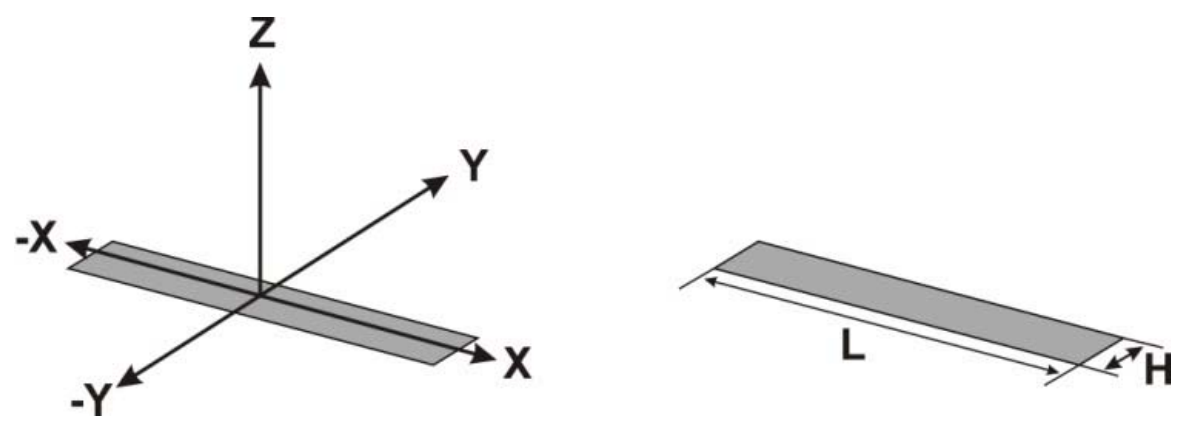

Figure 9: Definition of slot coordinate system and dimensions 


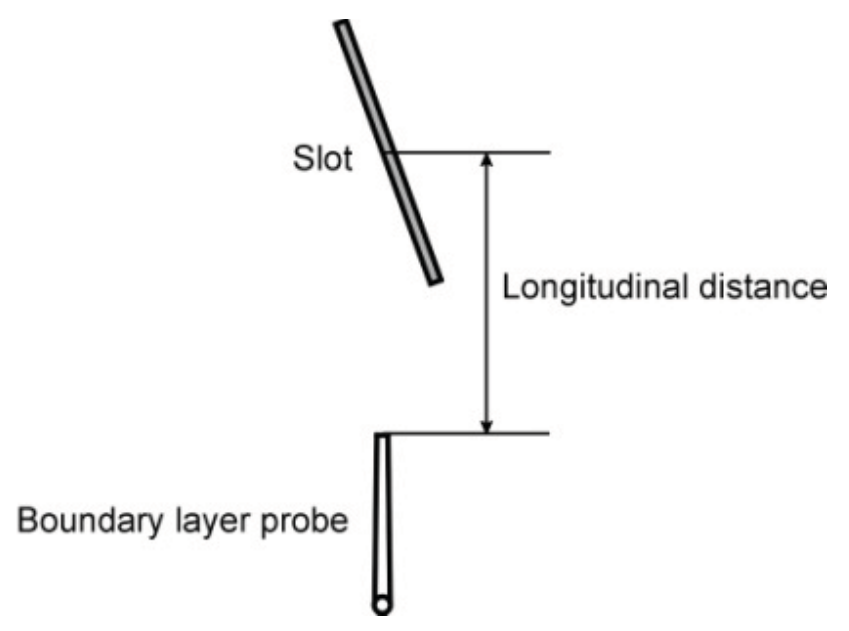

Figure 10: Definition of longitudinal distance $(X)$ between slot centre and boundary layer probe [plan view]

\section{Presentation and Discussion of Results}

\section{Effect of slot yaw angle $(\beta)$ and longitudinal distance $(X)$}

The effect of $\beta$ on the downstream boundary layer velocity profile was investigated. The time-averaged freestream velocity $(U)$ was constant for all tests, is $8.36 \mathrm{~m} / \mathrm{s}$ and the resulting $C_{\mu}$ is $1.01 \times 10^{-4}$. Since the impact of synthetic jet is clear in laminar boundary layer, the lowest average freestream velocity available $(8.36 \mathrm{~m} / \mathrm{s})$ is applied for this test to ensure laminar boundary layer. The $\beta$ varied from $0^{\circ}$ to $90^{\circ}$.

Boundary layer velocity profiles in the downstream flowfield are presented in Figure 11. The SJG has an effect on the boundary layer velocity profile up to $X=400 \mathrm{~mm}$. With increase in longitudinal distance $(X)$, the effect of synthetic jet and the effect of slot yaw angle change are diminished. Therefore, the proper longitudinal distance between the SJG and flow separation point is determined to be $20 \mathrm{~mm} \sim 100 \mathrm{~mm}$ in this case. $(\mathrm{X} / \mathrm{H}=$ $26.7 \sim \mathrm{X} / \mathrm{H}=133.3$ ) For easy comparison, laminar and turbulent boundary layer velocity profiles by empirical equations are included on each plot and the time-averaged nondimensional velocity profiles are compared with these turbulent and laminar velocity profile approximations. The lateral position of boundary layer probe on the plate is constant as $\mathrm{Y}=0 \mathrm{~mm}(\mathrm{Y} / \mathrm{H}=0)$. The boundary layer velocity profile with $\mathrm{SJG}$-off condition termed as baseline velocity profile. Since the one of the primary objective of this study is identifying the optimum- $\beta$ to achieve turbulent boundary layer, the $\beta$, which result in the closest boundary layer to turbulent approximation, will be considered as the optimum amount. 


\section{$X=20 \mathrm{~mm}(X / H=26.7)$; refer to Figure 11(a)}

The baseline velocity profile (SJG-off) is very close to the theoretical laminar boundary layer approximation. Following excitation of the SJG, there is a dramatic change in the boundary layer velocity profile. With $\beta=0^{\circ}$ and $\beta=10^{\circ}$, the velocity profile is distorted and forms a clear "low-speed core". It is postulated that these velocity profile distortions are due to the presence of counter-rotating vortex pair (CVP) over the slot and are consistent with the studies of both Bridges et $\mathrm{al}^{[2]}$ and Zhang et $\mathrm{al}^{[1]}$. With $\beta=20^{\circ}$, the velocity profile is the closest to the turbulent $1 / 7$ th power law approximation. For values of $\beta>20^{\circ}$, the impact of the SJG is diminished. For $\beta=40^{\circ} \sim 90^{\circ}$, the impact of $\beta$ in the velocity profile is negligible, but the synthetic jet results in a fuller boundary layer velocity profile than the undisturbed plate (SJG-off). When $\beta=0^{\circ}$, the centre of the lowspeed core is located at $Z / \delta=0.65$ and the relative velocity $(u / U)$ of the core is 0.7 . The position of the core is moved downward to $Z / \delta=0.25$ when the $\beta$ is increased to $\beta=10^{\circ}$ and the relative velocity in the core reduced further to $u / U=0.63$. If $\beta$ is above $20^{\circ}$, lowspeed core structure disappears and the boundary layer velocity profile is closest to turbulent approximation with $\beta=20^{\circ}$. In case of $\beta>20^{\circ}$, velocity profiles move away from the turbulent approximation. Since the target of synthetic jet application is transforming laminar boundary layer to turbulent one, $\beta=20^{\circ}$ is determined as the optimised- $\beta$ at this condition.

\section{$X=50 \mathrm{~mm}(X / H=66.7)$; refer to Figure 11(b)}

Similar to the result at $X=20 \mathrm{~mm}$, a slot yaw angle $\beta<20^{\circ}$ results in a distortion of velocity profile (the appearance of a low-speed core). The boundary layer profile is closest to turbulent approximation with $\beta=20^{\circ}$. If $\beta>20^{\circ}$, the $\beta$ makes the velocity profile moves away from the turbulent velocity profile approximation. That is to say, $\beta>20^{\circ}$ is less effective for velocity profile modification than $\beta=20^{\circ}$. The diameter of the low-speed core is increased and the relative velocity in the core is increased compared to that at $X=20 \mathrm{~mm}$, this is assumed to be due to the diffusion of the low-speed core. With $20^{\circ}<\beta<30^{\circ}$, the velocity profile becomes closer to turbulent approximation than that of $X=20 \mathrm{~mm}$. The velocity profiles with $0^{\circ}<\beta<20^{\circ}$ present that the low-speed core structure due to vortex pair is breaking down by increasing of $\beta$. In view of achieving turbulent boundary layer, author is considering the low-speed core structure is not much helpful.

\section{$\mathrm{X}=100 \mathrm{~mm}(\mathrm{X} / \mathrm{H}=133.3)$; refer to Figure 11(c)}

The baseline boundary layer velocity profile from $X=100 \mathrm{~mm}$ have fundamental differences with those of upstream positions. Due to the imperfection of wind tunnel model fabrication, a small step, which is lower than $0.5 \mathrm{~mm}$, was appeared at $X=75 \mathrm{~mm}$ (The turntable boundary) and it spoiled the downstream velocity profiles. The velocity profiles inside turntable (before step) are agreed with no-slip condition. However the velocity profiles outside turntable (after step) violate the no-slip condition. Therefore, the baseline velocity profiles after $X=75 \mathrm{~mm}$ do not agree with theory. In case of baseline velocity profile, the first data point from the surface is out of the velocity profile curve and it is postulated to be happened by the forward facing step on the boundary of 
turntable. When $\beta=5^{\circ} \sim 15^{\circ}$, the low-speed cores are apparent with a consequence distortion of the velocity profiles, such that the lower part of velocity profile overshoots the turbulent boundary layer approximation. As a result of an increase of the slot yaw angle $(\beta)$, the velocity profile becomes more like the turbulent boundary layer velocity profile approximation. As for the previous result, the velocity profile is closest to the turbulent approximation with $\beta=20^{\circ}$. If $\beta>20^{\circ}$, the effectiveness of synthetic jet is diminished. However, the synthetic jet causes a fuller velocity profile than the flat plate baseline (SJG-off condition) regardless of $\beta$.

When $\beta=0^{\circ}$, boundary layer is not affected by CVP. This may be due to the displacement of the CVP wake structure, which is drifting laterally in the $+Y$ direction, while the velocity profile is measured at $\mathrm{Y} / \mathrm{H}=0$. In the case of $5^{\circ} \leq \beta<20^{\circ}$, a weak wake structure is still detected, the velocity profile appears fully turbulent when $\beta=20^{\circ}$. Increasing $\beta$ above $20^{\circ}$ results in a reduction of synthetic jet impact on the boundary layer. However, every slot angle above $\beta=20^{\circ}$ still resulted in a fuller boundary layer velocity profile than SJG-off condition.

\section{$X=150 \mathrm{~mm}(X / H=200.0)$; refer to Figure 11(d)}

Because the low-speed core structure shifted to spanwise direction $(+Y)$, it does not appeared from $X=150 \mathrm{~mm}(\mathrm{Y} / \mathrm{H}=0)$. In the event of $\beta=5^{\circ} \sim 20^{\circ}$, the velocity profile keep approaching to the turbulent approximation. Like the previous, more forward position, a fully turbulent velocity profile is apparent when $\beta=20^{\circ}$ and increasing $\beta$ beyond $20^{\circ}$ does not improve the profile. Therefore $\beta=20^{\circ}$ is identified as the optimum- $\beta$ for flow separation control at this condition.

\section{$X=200 \mathrm{~mm}(X / H=266.7)$; refer to Figure 11(e)}

Like $X=150 \mathrm{~mm}$, the velocity profile approached to turbulent approximation up to $\beta=20^{\circ}$. The application of synthetic jet transforms the boundary layer from laminar to turbulent regardless of $\beta$. Though SJG results in fuller boundary layer velocity profile, the effect of SJG and change of $\beta$ does not result in as much change as upstream region $(X<200 \mathrm{~mm})$. Therefore the impact of $\beta$ is reduced at $X=200 \mathrm{~mm}$.

\section{$X=250 \mathrm{~mm}(\mathrm{X} / \mathrm{H}=\mathbf{3 3 3 . 3})$; refer to Figure 8-19(f)}

The baseline velocity profile is still laminar and the synthetic jet transforms it to turbulent. Like $X=200 \mathrm{~mm}$, the change of $\beta$ does not have much impact on velocity profiles. In case of SJG-on, the boundary layer velocity profile is concentrated in the narrow area regardless of $\beta$. If the SJG is not close to flow separation point, the impact of $\beta$ may not being significant.

\section{$X=300 \mathrm{~mm}(X / H=400.0)$; refer to Figure $11(\mathrm{~g})$}

The stethoscope measurement result presents that the laminar-turbulent transition appeared around $X=300 \mathrm{~mm}$. However, the baseline velocity profile is appeared as still 
laminar. By the application of synthetic jet, the velocity profile becomes very close to turbulent approximation.

\section{$X=400 \mathrm{~mm}(X / H=533.3)$; refer to Figure $11(\mathrm{~h})$}

Though the baseline velocity profile does not exactly agree with turbulent approximation, it is already turbulent (This judgement is based on stethoscope measurement). Therefore SJG was able to demonstrate only limited impact on velocity profile. The $\beta$ does not have significant impact.
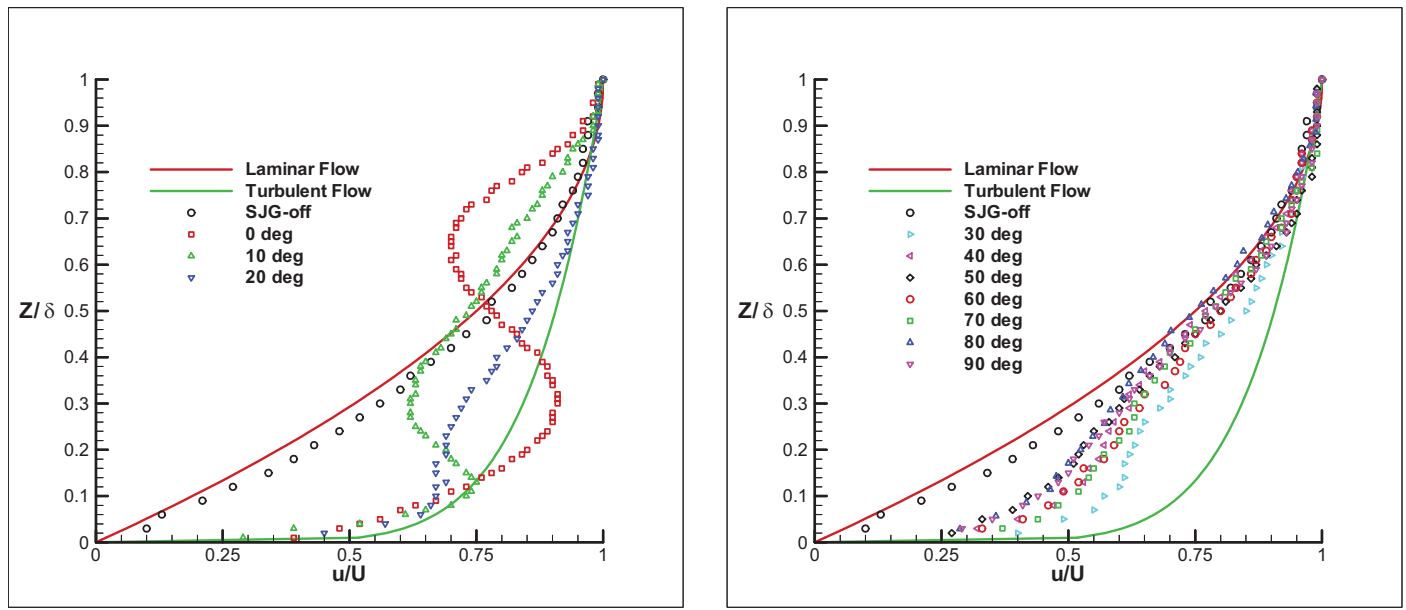

(a) $X=20 \mathrm{~mm}, R e_{x}=1.54 \times 10^{5}$
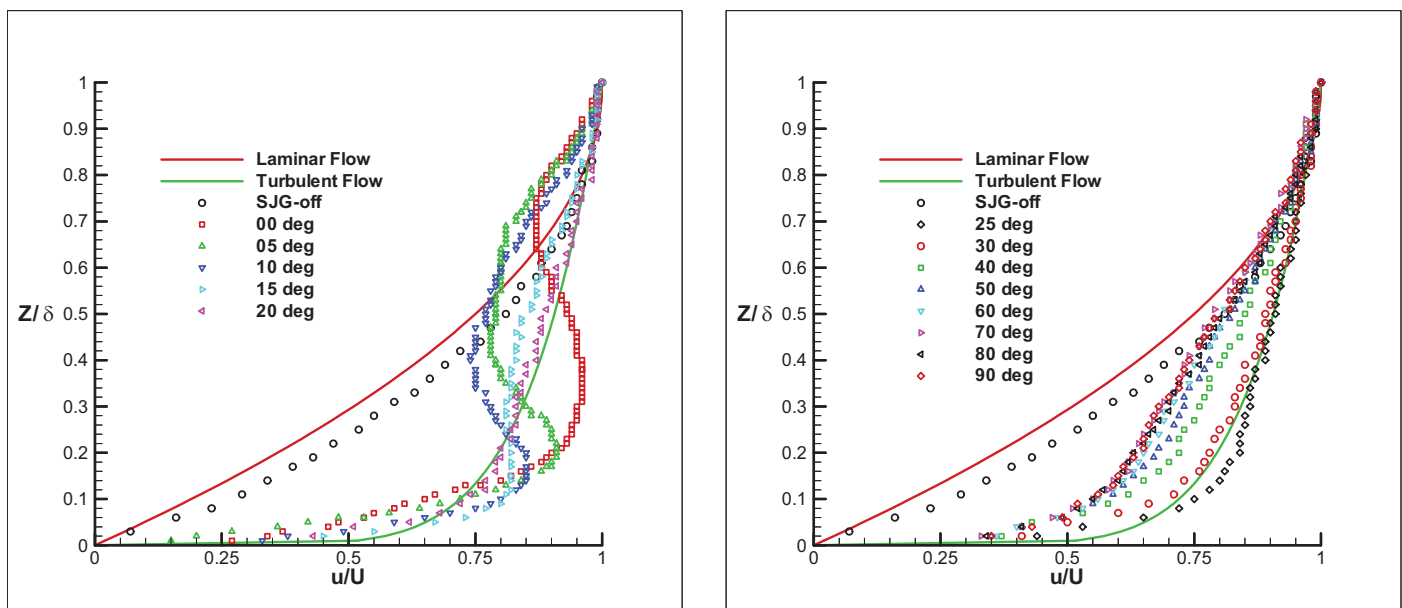

(b) $X=50 \mathrm{~mm}, R e_{x}=1.72 \times 10^{5}$

Figure 11: Time-averaged boundary layer velocity profile $\left(U=8.36 \mathrm{~m} / \mathrm{s}, C_{\mu}=1.01 \times 10^{-4}, V R=0.43\right)$ 

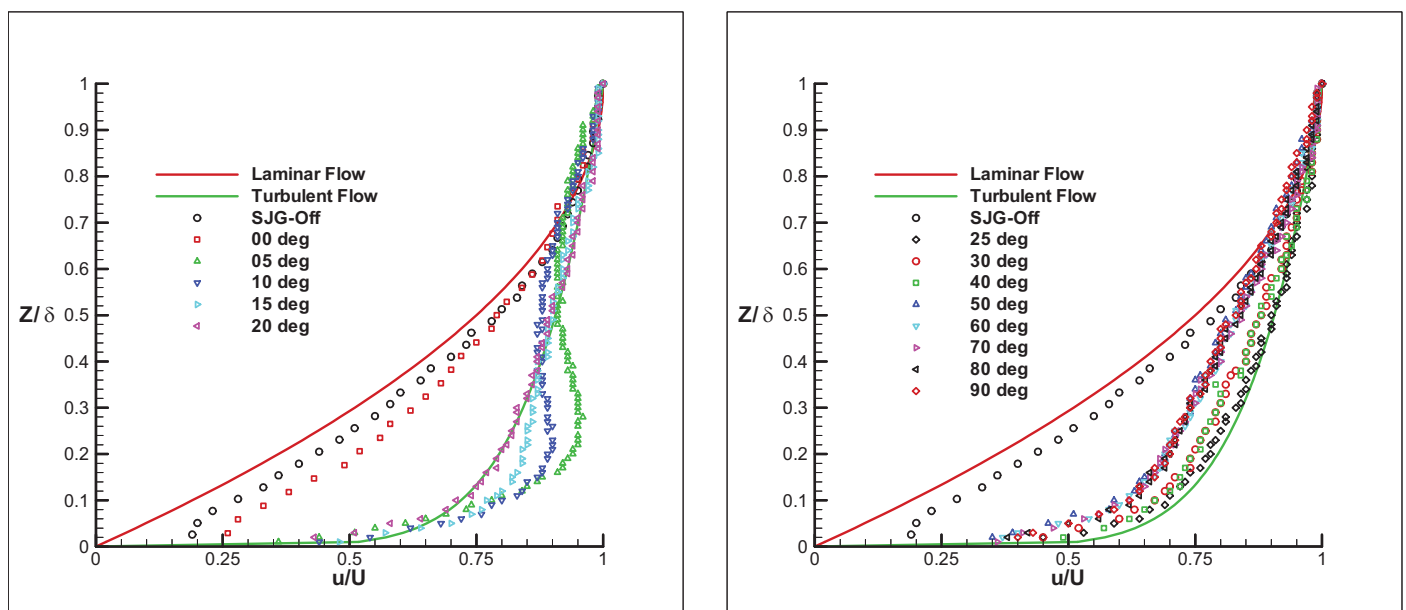

(c) $X=100 \mathrm{~mm}, R e_{X}=2.00 \times 10^{5}$
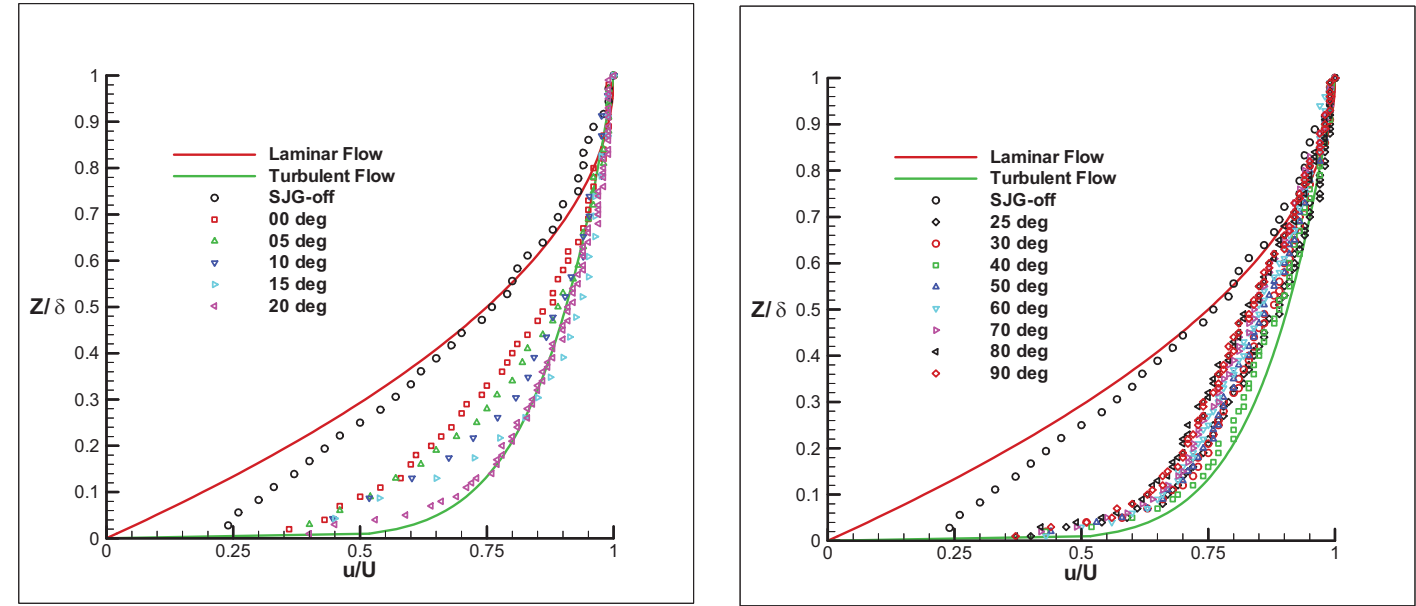

(d) $X=150 \mathrm{~mm}, R e_{x}=2.29 \times 10^{5}$
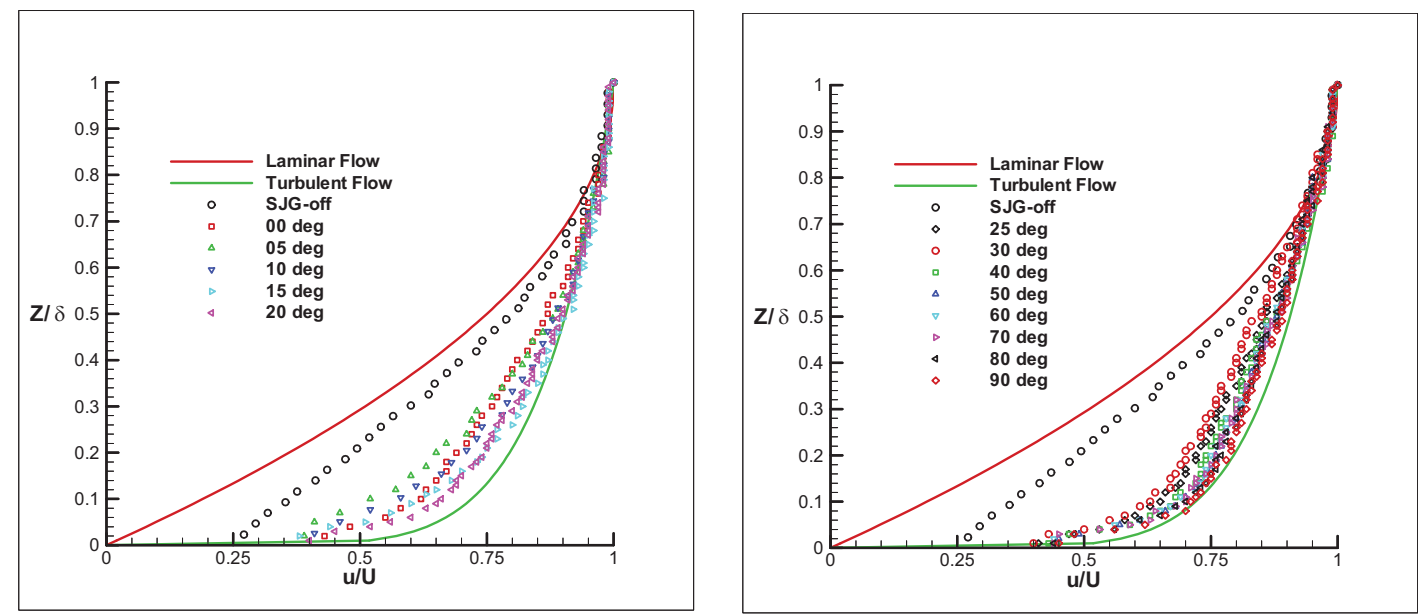

(e) $X=200 \mathrm{~mm}, R e_{x}=2.57 \times 10^{5}$

(Continued) Figure 11: Time-averaged boundary layer velocity profile

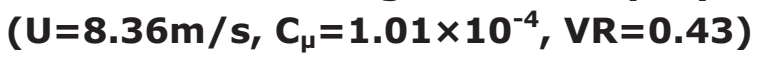



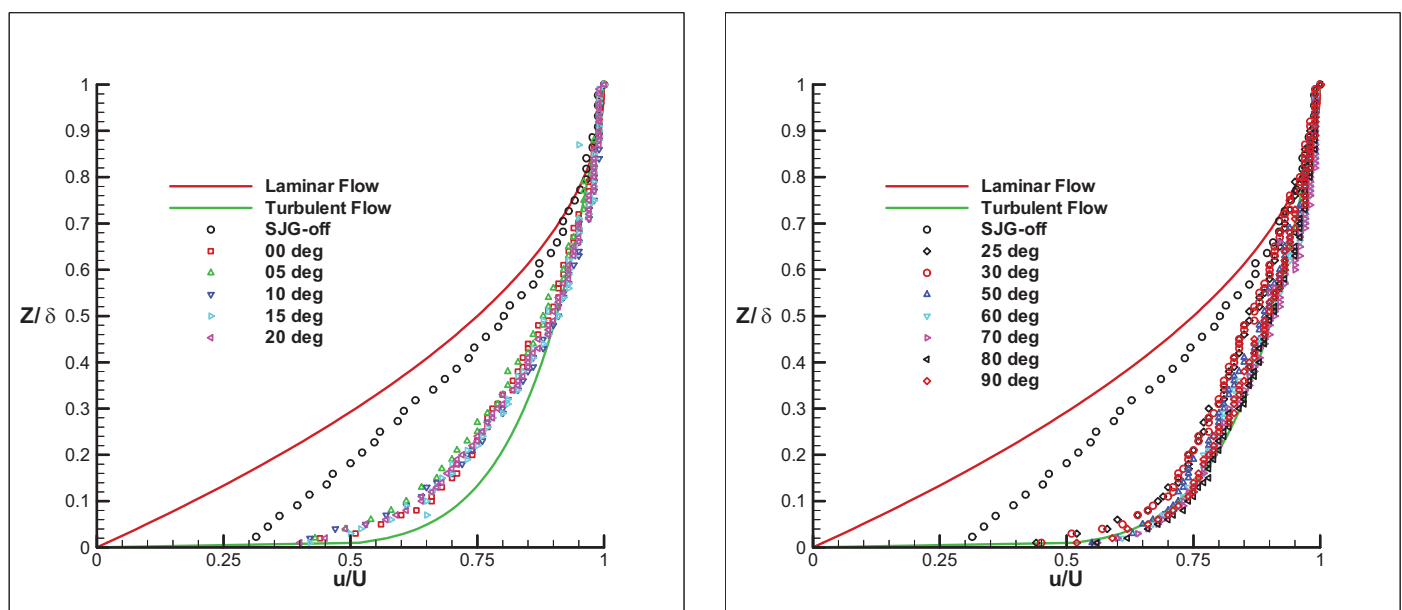

(f) $X=250 \mathrm{~mm}, R e_{x}=2.86 \times 10^{5}$
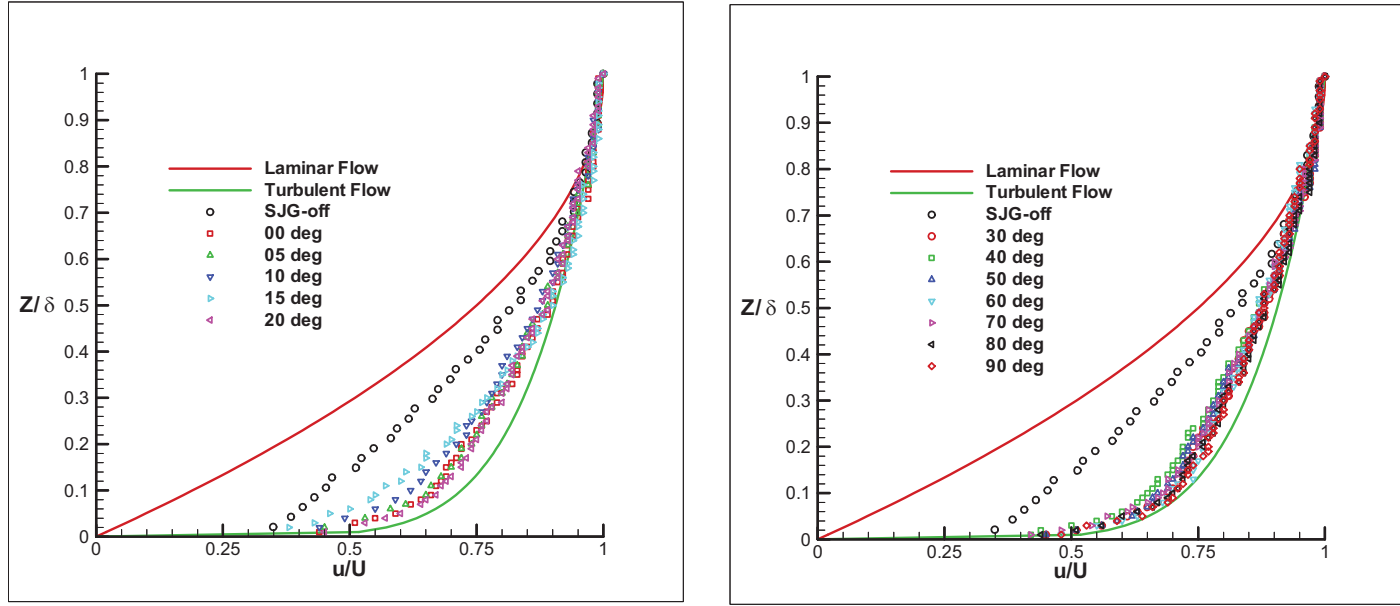

(g) $X=300 \mathrm{~mm}, R e_{x}=3.14 \times 10^{5}$
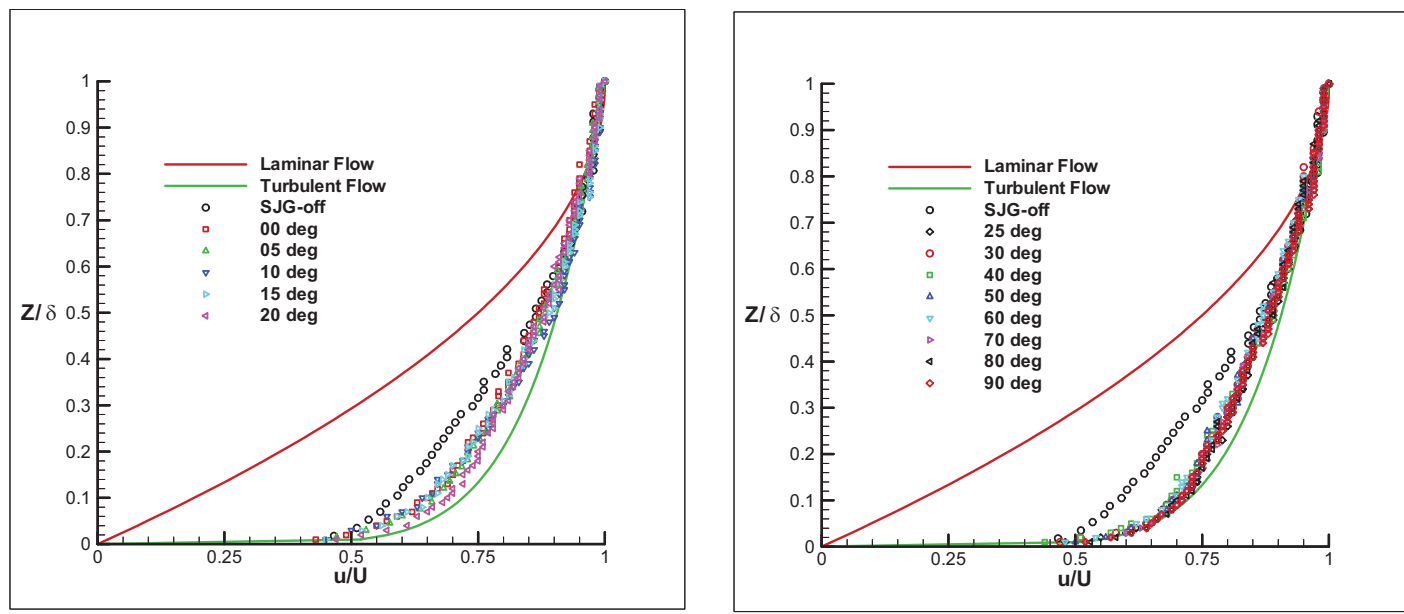

(h) $X=400 \mathrm{~mm}, R e_{x}=3.72 \times 10^{5}$

(Continued) Figure 11: Time-averaged boundary layer velocity profile $\left(U=8.36 \mathrm{~m} / \mathrm{s}, C_{\mu}=1.01 \times 10^{-4}, V R=0.43\right)$ 


\section{Detailed investigation of velocity defect (Low-speed core)}

The vortices are formed at the edge of the synthetic jet generator (SJG) orifice during the blowing stroke. In the case of a circular orifice, a vortex ring forms and if the orifice shape is a long aspect ratio slot, a CVP is created over the slot exit. ${ }^{[3]}$ According to the study of Compton et $\mathrm{al}{ }^{[4]}$ the skew and pitch angles of a continuous circular jet in a crossflow affect the strength and the rotating direction of resulting vortices. The effect of skew and pitch angle of synthetic jet with circular orifice may not have much difference from that of a steady jet with circular orifice. In the case of a synthetic jet with long aspect ratio slot, $\beta$ is expected to affect the strength and the rotating direction of its resulting vortices. Figure $\mathbf{1 2}$ presents the distortion of velocity profile due to the lowspeed core.

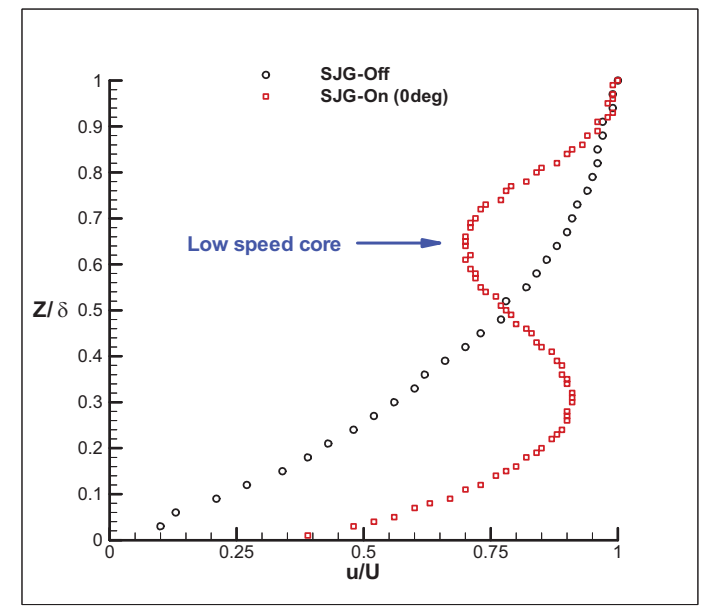

Figure 12: Time-averaged velocity profile change by the presence of CVP $\left(X=20 \mathrm{~mm}, U=8.36 \mathrm{~m} / \mathrm{s}, C_{\mu}=1.01 \times 10^{-4}, V R=0.43, R_{x}=1.54 \times 10^{5}\right)$

Figure 13 illustrates the schematic flowfield for synthetic jets with different $\beta\left(\beta=0^{\circ}\right.$, $\left.\beta=20^{\circ}, \beta=90^{\circ}\right)$. In the event of the slot being inline with free stream direction $\left(\beta=0^{\circ}\right)$, the least slot area is exposed to the crossflow and the resulting CVP arranged inline with freestream direction. In this condition, the CVP generates the strongest blockage effect downstream and its wake structure (CVP wake) will be appeared in the downstream flowfield. With increasing of $\beta$, a wider area of slot is exposed to crossflow and generates a single vortex structure similar to a continuous rectangular jet. When $\beta=90^{\circ}$, the synthetic jet presents the largest frontal area to the crossflow and causes the widest blockage effect on the boundary layer. However, the intensity of blockage effect with $\beta=90^{\circ}$ is not as strong as $\beta=0^{\circ}$. The data present the existence of CVP with $\beta=0^{\circ}$. However, CVP is appeared to be break down with higher $\beta\left(\beta>15^{\circ}\right)$, see Figure 11 . Therefore the CVPs in Figure 13(b) and (c) are expected to be destroyed by crossflow. The strength of blockage effect depends on $\mathrm{C}_{\mu}$ and the streamwise vortex structure does not appear with $\beta=90^{\circ}$. Some researchers have applied a synthetic jet with a long spanwise slot $\left(\beta=90^{\circ}\right)$ for virtual aeroshaping. ${ }^{[5]}$ The configuration of Figure $1(c)$ is important for those cases. 


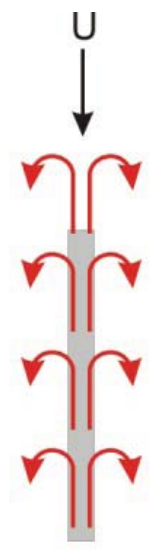

(a) $\beta=0^{\circ}$

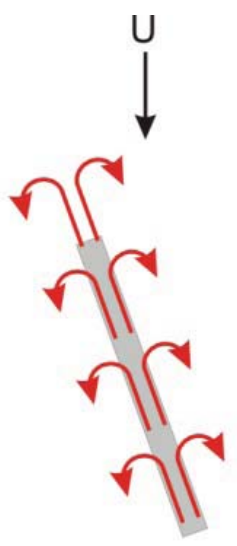

(b) $\beta=20^{\circ}$
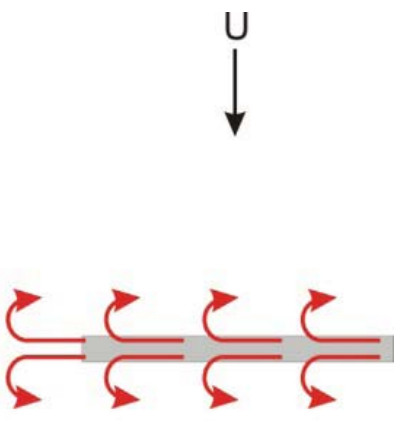

(c) $\beta=90^{\circ}$

\section{CVP Streamwise vortex Blockage effect}

\section{Figure 13: Counter-rotating Vortex Pair (CVP) in the crossflow}

\section{u/U distribution analysis in the downstream flowfield of synthetic jet}

The non-dimensional velocity $(u / U)$ distribution in the downstream flowfield of the SJG was investigated. At $X=50 \mathrm{~mm}$, the mean freestream velocity $(U)$ was constant at 8.36 $\mathrm{m} / \mathrm{s}$, while $\beta$ varies from $0^{\circ}$ to $90^{\circ}$. The boundary layer probe traversed in a spanwise (lateral) direction from $\mathrm{Y}=-20 \mathrm{~mm}(\mathrm{Y} / \mathrm{H}=26.67)$ to $\mathrm{Y}=20 \mathrm{~mm}(\mathrm{Y} / \mathrm{H}=-26.67)$. The vertical traverse was from $Z=0.1 \mathrm{~mm}(Z / H=0.13)$ to $Z=15 \mathrm{~mm}(Z / H=20)$. The probe position increments $0.5 \mathrm{~mm}$ in the vertical direction $(\mathrm{Z} / \mathrm{H}=0.67)$ and $0.5 \mathrm{~mm} / 1 \mathrm{~mm}$ in the transverse direction $(\mathrm{Y} / \mathrm{H}=0.67 / 1.33)$. The data sampling frequency is $3 \mathrm{KHz}$ and the sampling time is 3 seconds. The lateral $(Y)$ and vertical ( $Z$ ) positions are nondimensionalised by the slot width $(\mathrm{H}=0.75 \mathrm{~mm})$. The nodes in Figure 14 represent the positions for data sampling. As can be seen, more concentrated nodes are arranged in the important area.

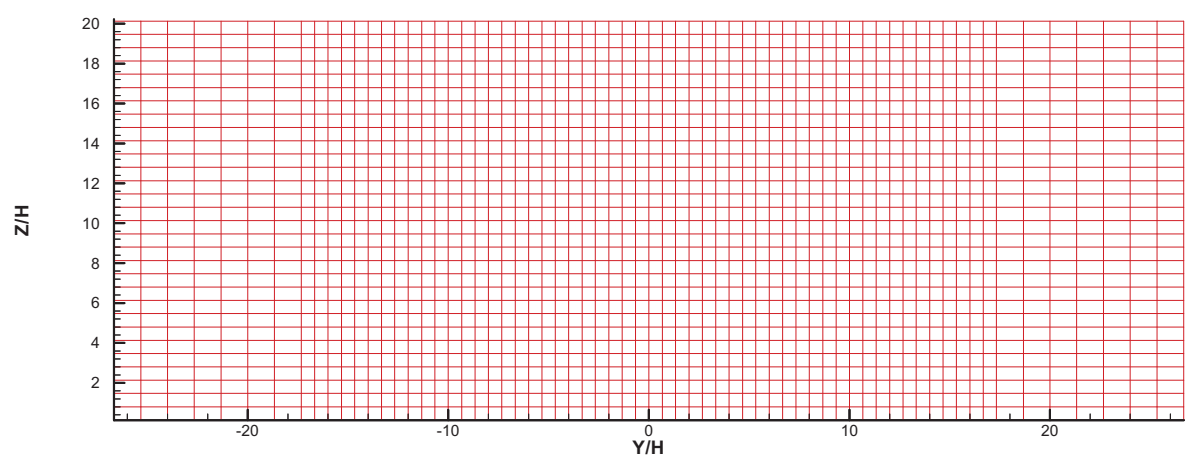

Figure 14: Traversing of boundary layer probe in $X-Y$ plane 


\section{Synthetic jet generator off (SJG-off), refer to Figure 15(a)}

The baseline non-dimensional velocity $(u / U)$ distribution shows constant distribution in transverse direction. The boundary layer extends up to $\mathrm{Z} / \mathrm{H}=4.4$.

\section{$\beta=0^{\circ}$, refer to Figure 15 (b)}

A clear counter rotating vortex (CVP) structure is appeared in the downstream flowfield, Though, there is no sufficient evidence to confirm the existence a CVP wake structure, also similar flow structures are reported by Bridges et $\mathrm{al}^{[2]}$ and Zaman et $\mathrm{al}^{[6]}$. Both studies applied a synthetic jet to a crossflow. Bridges et al used long aspect ratio slot and Zaman et al used circular orifice. The study of Zaman et al supports the proposed wake structure as that of a CVP. They presented streamwise mean velocity contours in the downstream flowfield. The mean velocity contour at $X / d=0.5(9.53 \mathrm{~mm})$ illustrates a CVP structure ( $d$ : orifice diameter). Their contour data at $X / d=5(95.25 \mathrm{~mm})$ presented a single circular structure which is similar with Figure 15(b). The CVP wake structure penetrates the boundary layer and increases the local boundary layer thickness. The height of the wake structure is more than $12 \mathrm{H}$ and the width of the wake is nominally $12 \mathrm{H}$. The lateral position of low-speed core is nominally at $\mathrm{Y} / \mathrm{H}=3$ and the nondimensional height of low-speed core is at nominally $\mathrm{Z} / \mathrm{H}=8(\mathrm{Z}=6 \mathrm{~mm})$. The core of the wake displaces to $\mathrm{Y} / \mathrm{H}=3$. Although the flat plate model is installed inline with wind tunnel test section, a small freestream yaw angle may cause drifting of the CVP core. This spanwise drifting is supposed to be caused by the instrument imperfection (sideslip of test section) and does not have engineering importance. The study of David et $\mathrm{al}^{[7]}$ gives more information about the CVP wake structure, in terms of velocity contour plots with velocity vectors. Their PIV measurement result presents two CVP structures at each side of low-speed core structure. Therefore, although the velocity contour in Figure 15(b) appears to be a single vortex structure, it is assumed to be caused by two counter rotating vortex pairs.

\section{$\beta=5^{\circ}$, refer to Figure $15(\mathrm{c})$}

The CVP wake begins to decay and is displaced laterally. The height of low-speed core is nominally $\mathrm{Z} / \mathrm{H}=7$. As a result of the increase in $\beta$, the low-speed core moved lower down and begins to merge into the boundary layer.

\section{$\beta=10^{\circ}$, refer to Figure 15 (d)}

The CVP wake is further broken down. The local boundary layer change takes place from $\mathrm{Y} / \mathrm{H}=-5$ to $\mathrm{Y} / \mathrm{H}=7$. The partially broken wake structure is asymmetric around $\mathrm{Y} / \mathrm{H}=0$. The low-speed core height is nominally $\mathrm{Z} / \mathrm{H}=4$ and this means the low-speed core begins to merge into the boundary layer. The height of the wake structure is $\mathrm{Z} / \mathrm{H}=10$.

\section{$\beta=15^{\circ}$, refer to Figure $15(\mathrm{e})$}

In the event of $\beta$ being more than $15^{\circ}$, a CVP wake structure no longer exists. Zhang et $\mathrm{al}^{[1]}$ generated a streamwise vortex structure using a rectangular steady jet with a pitch (a) and yaw angle $(\beta)$. The relative velocity contour $(u / U)$ in the down stream flowfield of 
steady jet $\left(\beta=45^{\circ}\right)$ is similar to the corresponding synthetic jet $\left(\beta=15^{\circ} \sim 20^{\circ}\right)$ Therefore it is postulated that a single streamwise vortex structure is produced by the synthetic jet. The boundary layer is thickest at the lateral location $\mathrm{Y} / \mathrm{H}=5$ and the synthetic jet affects the boundary layer from $\mathrm{Y} / \mathrm{H}=-6$ to $\mathrm{Y} / \mathrm{H}=10$.

\section{$\boldsymbol{\beta}=\mathbf{2 0 ^ { \circ }}$, refer to Figure $\mathbf{1 5 ( f )}$}

A streamwise vortex structure continues to be apparent and the synthetic jet affects a wider area. In general terms, the synthetic jet affects from $Y / H=-6$ to $Y / H=11$. Although it is not possible to prove the existence of secondary vortex from this data, the author expects there to be a small secondary vortex structure on the left side of vortex core.

$\beta=30^{\circ}, 50^{\circ}, 70^{\circ}, 90^{\circ}$, refer to Figure 15(g) (j)

The increase of $\beta$ means the synthetic jet affects a wider area. This phenomenon takes place due to the increase of projected slot area to the free stream. However, boundary layer control effectiveness is relatively poor compared to lower- $\beta$ condition $\left(\beta \leq 25^{\circ}\right)$. Because wider area is exposed to crossflow, the wider blockage effect was expected. The strength of the blockage effect depends on $\mathrm{C}_{\mu}$.

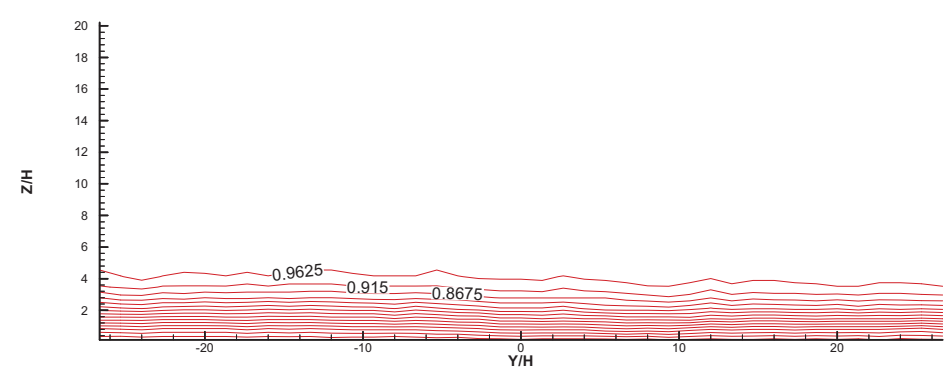

(a) SJG-off

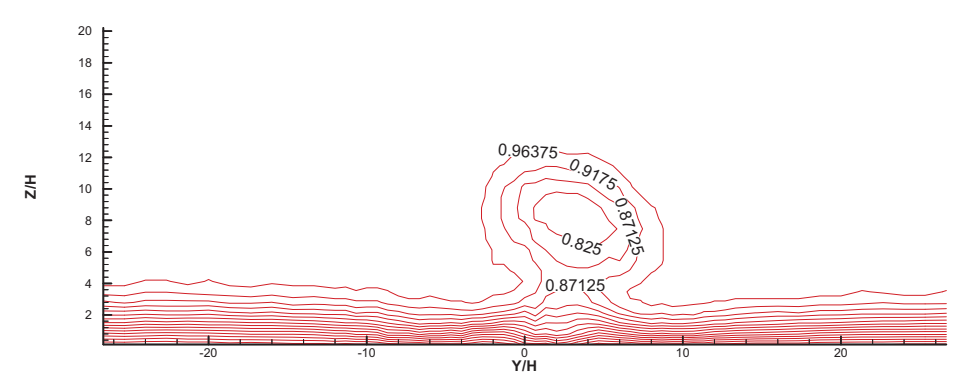

(b) $\beta=0^{\circ}$

Figure 15: Relative velocity (u/U) contours by synthetic jet $\left(\operatorname{Re}_{\mathrm{x}}=1.72 \times 105, C_{\mu}=1.01 \times 10-4, V R=0.43, U=8.36 \mathrm{~m} / \mathrm{s}\right)$ 


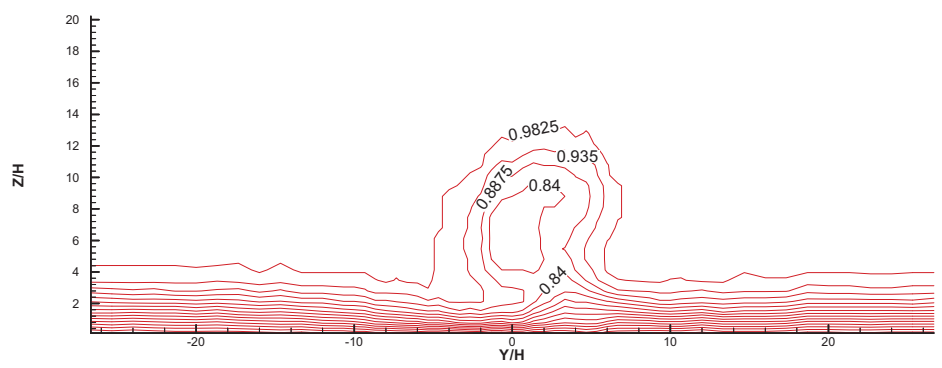

(c) $\beta=5^{\circ}$

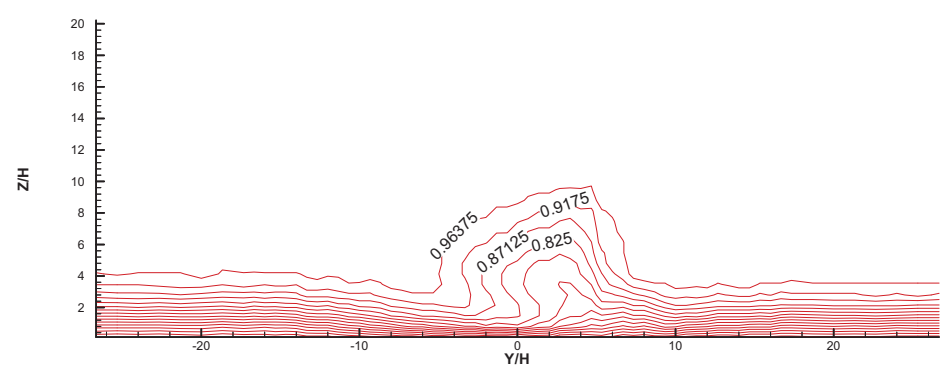

(d) $\beta=10^{\circ}$

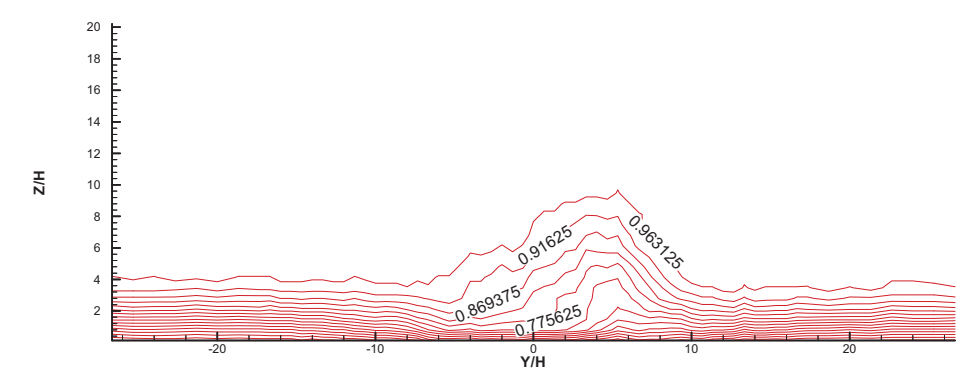

(e) $\beta=15^{\circ}$

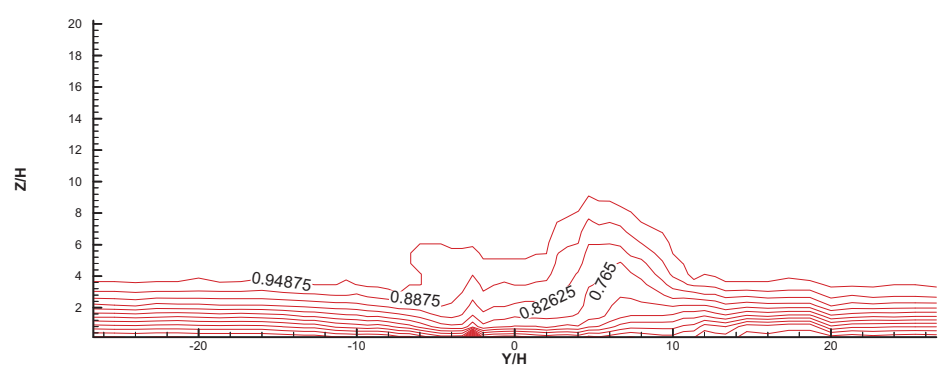

(f) $\beta=20^{\circ}$

Figure 15: Relative velocity (u/U) contours by synthetic jet $\left(R_{x}=1.72 \times 105, C_{\mu}=1.01 \times 10-4, V R=0.43, U=8.36 \mathrm{~m} / \mathrm{s}\right)$ 


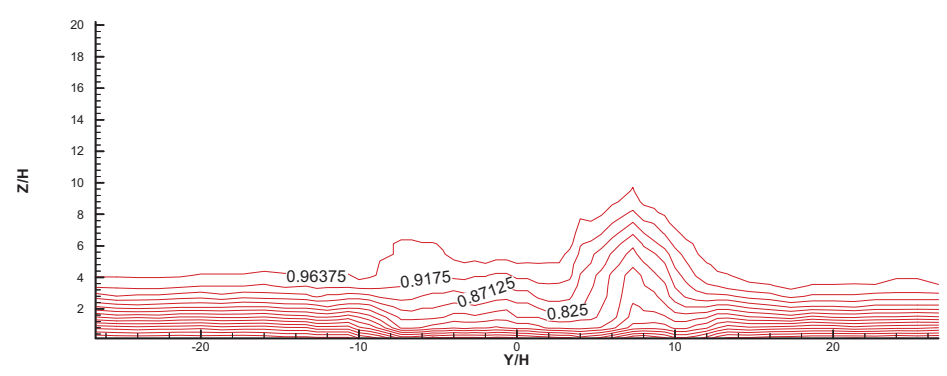

(g) $\beta=30^{\circ}$

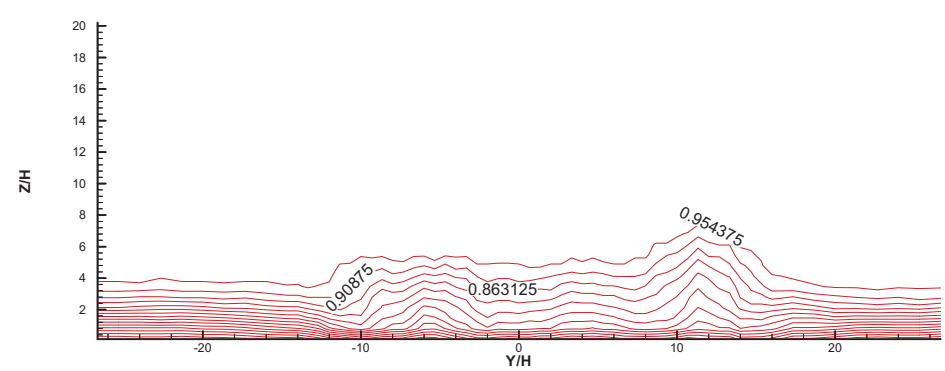

(h) $\beta=50^{\circ}$

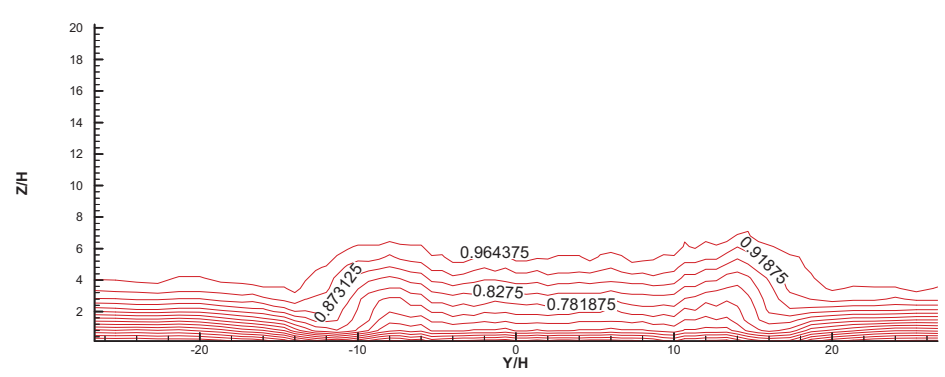

(i) $\beta=70^{\circ}$

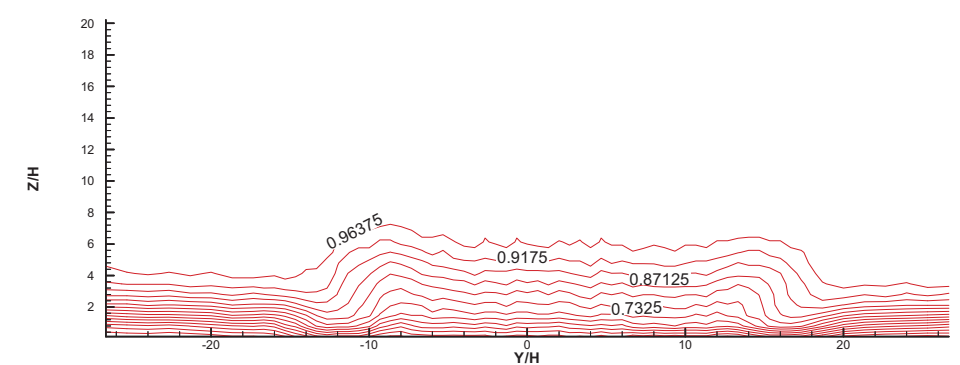

(j) $\beta=90^{\circ}$

Figure 15: Relative velocity (u/U) contours by synthetic jet $\left(\operatorname{Re}_{\mathrm{x}}=1.72 \times 105, C_{\mu}=1.01 \times 10-4, V R=0.43, U=8.36 \mathrm{~m} / \mathrm{s}\right)$ 


\section{Conclusions}

The flow phenomenon in the downstream flowfield of synthetic jet strongly depends on the Reynolds number, the intensity of synthetic jet relative to cross flow $\left(C_{\mu}, V R\right)$ and slot yaw angle $(\beta)$. In case of low $\beta\left(\beta<20^{\circ}\right)$, synthetic jet caused a low speed core in the downstream boundary layer. This low speed core is created by the counter rotating vortex pair (CVP) due to synthetic jet. By increasing synthetic jet yaw angle $\left(\beta \geq 20^{\circ}\right)$ the downstream flowfield structure is fundamentally changed. The synthetic jet results in a single streamwise vortex which provides higher momentum to the downstream flowfield. If the slot yaw angle increased further, synthetic jet does not create a streamwise vortex and just forms a blockage effect in the wider area. Synthetic jet triggers laminarturbulent transition. Therefore synthetic is able to work as an active turbulator.

The effectiveness of synthetic jet generator is reduced by increasing the longitudinal distance from the centre of the slot $(X)$. In the view point of boundary layer velocity profile modification, synthetic jet generator is most effective when the longitudinal distance to the flow separation point from SJG $(X)$ is $50 \mathrm{~mm}(X / H=66.67) \sim 150 \mathrm{~mm}$ $(\mathrm{X} / \mathrm{H}=200)$.

The slot yaw angle is one of the decisive parameters for the flow control by synthetic jet and the boundary layer velocity profile is closest to the turbulent velocity profile approximation with $\beta=20^{\circ}$. The low speed core structure is generated by the interaction of synthetic jet and freestream (cross flow). This wake structure is most clear by $\beta=0^{\circ}$. 


\section{RefERENCES}

[1] Xin zhang et al: Measurements of a longitudinal vortex generated by a rectangular jet in a turbulent boundary layer, Phys. Fluid Vol. 9, No. 6, 1997

[2] Bridge Amanda et al: The influence of orifice orientation on the integration of a synthetic jet with a turbulent boundary layer, AIAA 2001-2774, June. 2001.

[3] Rerard E. Welch et al: Application of Harmonic Balance Technique to Synthetic Jet in Cross-Flow, AIAA 2005-1111, Jan. 2005

[4] Compton Debora A. et al: Streamwise vortex production by pitched and skewed jets in a turbulent boundary layer, AIAA J. Vol. 30, No.3, Mar. 1992

[5] PZT application manual, Murata Manufacturing Co., Ltd.

[6] K.B.M.Q. Zaman et al: Synthetic Jets in Cross-Flow. PART I: ROUND JET, AIAA 2003-3714, June.2003. Orlando. Florida.

[7] L.David et al: Cross Flow Investigation by Stereoscopic PIV Measurements, Laboratoire d'Etudes Aerodynamiques (UMR 6609-CNRS)

[8] Fang-Jenq Chen et al: Virtual Shaping of a Two-dimensional NACA 0015 Airfoil Using Synthetic Jet Acutuator, AIAA 2002-3273, 2002

[9] Conrad Y. Lee et al: Two-dimensional synthetic jet simulation,AIAA 2000-0406, Jan 2000

[10] Yair guy et al; Velocity measurements in a synthetic jet, AIAA 2001-0118, Jan 2001

[11] Ari Glezer: The formation of vortex rings, Phys. Fluid, Vol. 31, No. 12

[12] Linda D. Kral: Active flow control technology

[13] J.Skinner et al: A Piezoelectric Membrane Generator for MEMS Power, AIAA 2002-5745, September 2002. 